\title{
The greatest prime function of real variable
}

\author{
Maher Nazmi Qarawani \\ Department of Mathematics, Alquds Open University, Palestine,West-Bank, Salfit P.O.Box 37 \\ E-mail: mkerawani@qou.edu
}

Copyright () 2015 Maher Nazmi Qarawani. This is an open access article distributed under the Creative Commons Attribution License, which permits unrestricted use, distribution, and reproduction in any medium, provided the original work is properly cited.

\begin{abstract}
In this paper, we introduce a new function $f(x)=[x]_{p}, x \in R$, with $\mathrm{x} \geq 2$, which we call the greatest prime function. In addition, we give an extension of the function to $R$, and then use this definition to prove some inequalities and properties of this function. Some illustrative examples are given.
\end{abstract}

Keywords: Greatest Prime Function; Gap Function; Inequalities.

\section{Introduction}

In this paper, we introduce a new function

$f(x)=[x]_{p}, x \in R$, with $x \geq 2$,

Which we will call the greatest prime function (or the floor prime function), where $[x]_{p}\left(\right.$ or $\left.\left\lfloor_{x}\right\rfloor_{p}\right)$ represents the greatest prime number less than or equal to the real number example, $[2.99]_{p}=2,[\sqrt{8}]_{p}=2,[7]_{p}=7,[10.87]_{p}=7$, $[\sqrt[3]{9578643}]_{p}=211$, etc.

The domain of the greatest prime function is $R \backslash(-\infty, 2)$ and its range is the set of all prime numbers. Note that the graph of the greatest prime function jumps one unit at the prime number 3 , two units at 5 , two units at 7 , and four units at 11 , and so on. This implies that the function is discontinuous at each prime number. The graph of $f(x)=[x]_{p}$, is shown in Fig.1.

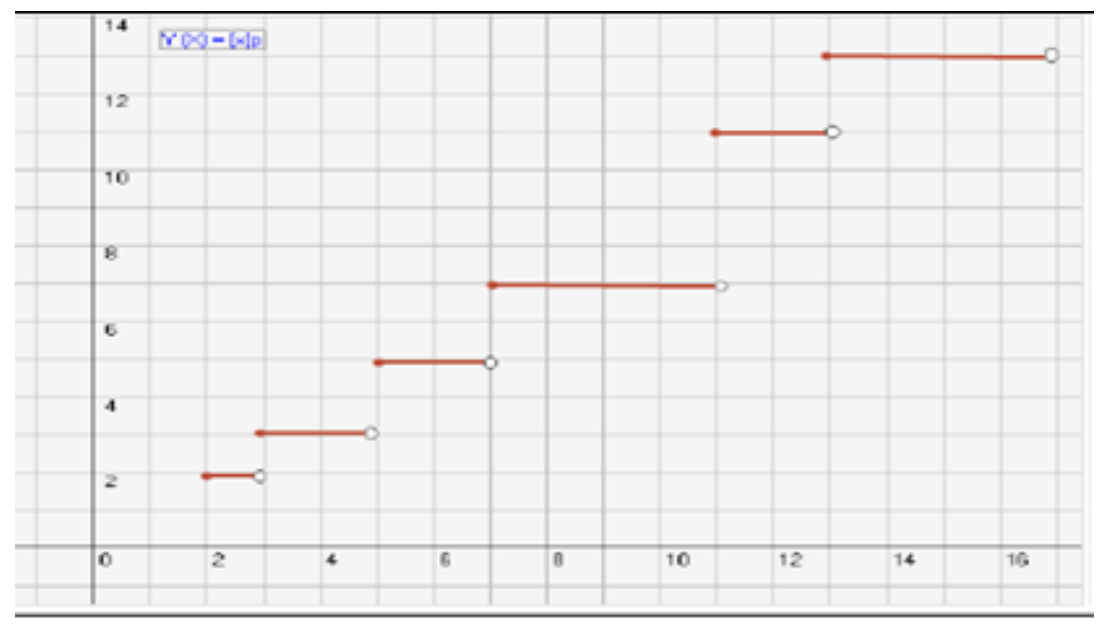

Fig. 1 
Here, we also define a fractional gap function by $\{x\}_{p}=x-[x]_{p}, \forall x \geq 2$, which means the non-integer gap number. The graph of $f(x)=x-[x]_{p}$ is shown in Fig.2.

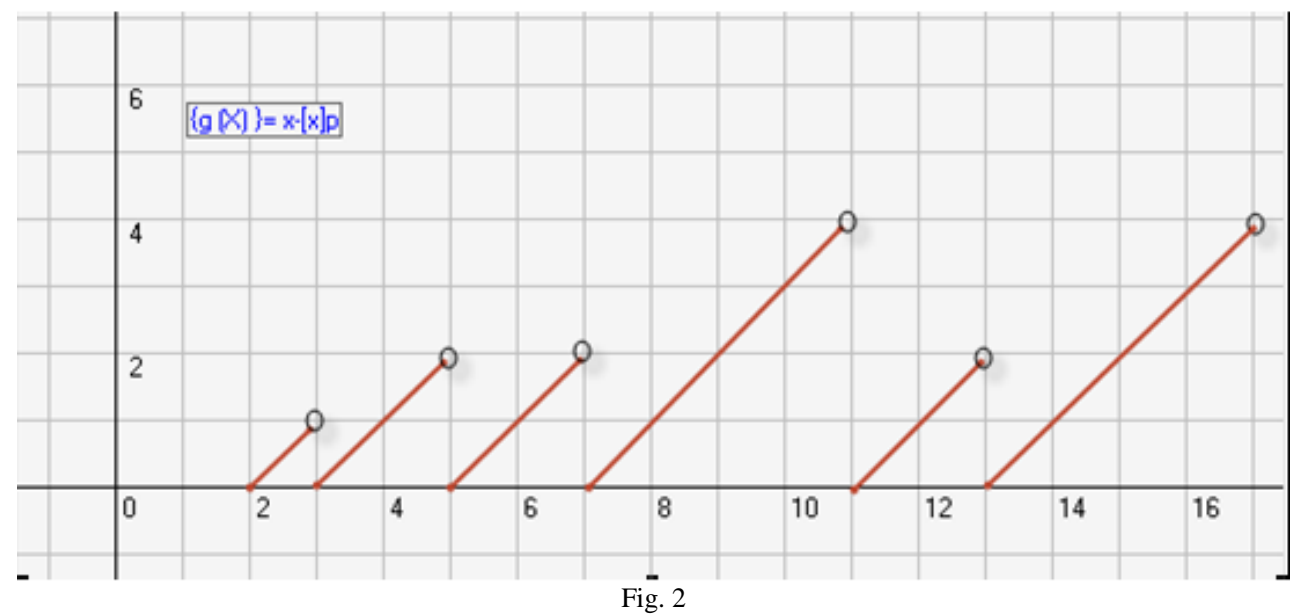

For example, if $x=(7479089197619)^{1 / 2}$ then

$$
\begin{aligned}
\{x\}_{p} & =\left\{(7479089197619)^{1 / 2}\right\}=(7479089197619)^{1 / 2}-\left[(7479089197619)^{1 / 2}\right]_{p} \\
& =2734792.35-2734763=29.35
\end{aligned}
$$

It should be noted that the first prime number next to the prime number 2734763 is 2734793 , hence the gap between them is 30, which is greater than the non-integer gap 29.35. In general, it follows from the definition of greatest prime function that $\{x\}_{p}<g_{n}$, where $g_{n}=p_{n+1}-p_{n}$, and $p_{n} \leq x$. Indeed, let $n \geq 1$ be an integer, and let $p_{n} \geq 2$ denote the nth prime number. Then, since $p_{n} \leq x<p_{n+1}$, we obtain $\{x\}_{p}=x-[x]_{p}=x-p_{n}<p_{n+1}-p_{n}$.

In this paper, we also extend the greatest prime function to $R$ in an odd manner,

$G_{p}(x)= \begin{cases}{[x]_{p},} & x \geq 2 \\ 0, & |x|<2 \\ -[-x]_{p}, & x \leq-2\end{cases}$

Indeed, the function $G_{p}(x)$ defined by (1) is an odd function:

$$
\begin{aligned}
& G_{p}(-x)= \begin{cases}{[-x]_{p},} & -x \geq 2 \\
0, & |x|<2 \\
-[x]_{p}, & -x \leq-2\end{cases} \\
& = \begin{cases}-[x]_{p}, & x \geq 2 \\
0, & |x|<2 \\
{[-x]_{p},} & x \leq-2\end{cases} \\
& =-G_{p}(x), \forall x \in R \text {. }
\end{aligned}
$$

For instance,

$$
\begin{aligned}
G_{p}(\sqrt[5]{9842178956}) & =[99.68]_{p}=97, G_{p}(-\sqrt[5]{9842178956}) \\
& =[-99.68]_{p}=-[99.68]_{p}=-97=-G_{p}(\sqrt[5]{9842178956})
\end{aligned}
$$


Remark 1.1: Note that one can make an extension for the greatest prime function to $R$, in even manner, so that

$$
G_{p}(x)= \begin{cases}{[x]_{p},} & x \geq 2 \\ 0, & |x|<2 \\ {[-x]_{p},} & x \leq-2\end{cases}
$$

Remark 1.2: We can similarly define the least prime function (or the ceiling prime function), and denote it by $\lceil x\rceil_{p}$, where $\lceil x\rceil_{p}$ represents the smallest prime number greater than or equal to the real number $x$. For example,

$\lceil 2\rceil_{p}=2,\lceil 2.01\rceil_{p}=3,\lceil\sqrt{10.65}\rceil_{p}=5$

and so on.

Properties of the Greatest Prime Function

1) The function $[x]_{p}=x$ if and only if $x$ is a prime number.

2) The function $[x]_{p}=p_{n}, n \in \mathrm{Z}^{+}$, if and only if $p_{n} \leq x<p_{n+1}$, where $p_{n}$ is the nth prime number.

3) The function $[x]_{p} \leq x$, for any real number $x$.

4) The function $f(x)=[x]_{p}$ is nondecreasing for all $x \in R \backslash(-\infty, 2)$, i.e., if $x_{1} \leq x_{2}$, then $\left[x_{1}\right]_{p} \leq\left[x_{2}\right]_{p}$.

5) The function $f(x)=[x]_{p}$ is discontinuous at all primes $p_{n+1}, n \in \mathrm{Z}^{+}$and continuous at all $x$ satisfying $p_{n} \leq x<p_{n+1}$, because $[x]_{p}=p_{n}$, for any $n \in \mathrm{Z}^{+}$, with $n \geq 1$.

6) The function $f(x)=[x]_{p}$ is right-continuous at all primes $p_{n}$, because $\lim _{x \rightarrow p_{n}+}[x]_{p}=p_{n}=f\left(p_{n}\right)=\left[p_{n}\right]$, for any $n \in \mathrm{Z}^{+}, n \geq 2$.

Example 1.1 Find the discontinuity points of $f(x)=x[x]_{p}, x \in R$.

We find that $x[x]_{p}=p_{n} x$, where $p_{n} \leq x<p_{n+1}, n \in Z^{+}$. Since $f\left(p_{n}\right)=p_{n}^{2}=\lim _{x \rightarrow p_{n}+} p_{n} x$, and $\lim _{x \rightarrow p_{n}-} x[x]_{p}=p_{n-1} p_{n}$, then $f(x)=[x]_{p}$ is discontinuous at the points $x=p_{n}$, for all integers $n \geq 2$.

Example 1.2 Consider the function

$f(x)=\left\{\begin{array}{l}x\left[\frac{1}{x}\right]_{p}, 0<x \leq \frac{1}{2} \\ 1, x=0\end{array}\right.$.

The function $f(x)$ is continuous in all intervals $\frac{1}{p_{n+1}}<x \leq \frac{1}{p_{n}}$, for any $n \in \mathrm{Z}^{+}, n \geq 1$. Moreover,

$f\left(\frac{1}{p_{n}}\right)=1, f\left(\frac{1}{p_{n}}+0\right)=\lim _{x \rightarrow \frac{1}{p_{n}}+0} x\left[\frac{1}{x}\right]_{p}=\frac{p_{n-1}}{p_{n}}$.

Therefore, the function is discontinuous at the points $x=\frac{1}{p_{n}}, n \in \mathrm{Z}^{+}$, with $n \geq 2$. Now, we will show that $f(x)$ is continuous at $x=0$. Indeed, For $x \in\left(\frac{1}{p_{n+1}}, \frac{1}{p_{n}}\right)$, we have

$\frac{p_{n}}{p_{n+1}} \leq x\left[\frac{1}{x}\right]_{p}<\frac{p_{n+1}}{p_{n}}, n \in \mathrm{Z}^{+}$.

By the prime number theorem (see [4]), we have $\lim _{n \rightarrow \infty} \frac{p_{n+1}}{p_{n}}=1$. Therefore, since as $n \rightarrow \infty, x \rightarrow 0+$, we obtain

$\lim _{x \rightarrow 0+} f(x)=\lim _{x \rightarrow 0+} x\left[\frac{1}{x}\right]_{p}=1=f(0)$.

Properties of the Fractional Semi-gap Function

1) The fractional semi-gap function $\{x\}_{p}=0$ if and only if $x$ is a prime number, i.e. $x=[x]_{p}$. 
2) The function $0 \leq\{x\}_{p}<g_{n}$, for all $x \in R, n \in \mathrm{Z}^{+}$, where $g_{n}=p_{n+1}-p_{n}, n \geq 1$.

3) The fractional semi-gap function $\{x\}_{p}$ is discontinuous at all prime $p_{n+1}, n \in \mathrm{Z}^{+}$since

$$
\lim _{x \rightarrow p_{n+1^{-}}}\{x\}_{p}=\lim _{x \rightarrow p_{n+1^{-}}}\left(x-[x]_{p}\right)=p_{n+1}-p_{n}
$$

and

$$
\lim _{x \rightarrow p_{n+1}+}\{x\}_{p}=\lim _{x \rightarrow p_{n+1}}\left(x-[x]_{p}\right)=\left\{p_{n+1}\right\}_{p}=0 .
$$

4) The function $\{x\}_{p}$ is right-continuous at all $x$ satisfying $p_{n} \leq x<p_{n+1}$, for any $n \in \mathrm{Z}^{+}$, with $n \geq 1$.

5) The function $\{x\}_{p}$ is continuous at all primes $p_{n}$, because $\lim _{x \rightarrow p_{n}+}\{x\}_{p}=0=\left\{p_{n}\right\}_{p}$, for any $n \in Z^{+}, n \geq 2$. Now, we will give the statement of an interesting conjecture that was first stated in 1982 by the Iranian mathematician Farideh Firoozbakht [3], which we will use to establish some inequalities.

Firoozbakht's Conjecture: If $p_{k}$ is the kth prime, then the sequence $\left(p_{k}\right)^{1 / k}$ is strictly decreasing. Equivalently, for all $k \geq 1$ we have $p_{k+1}^{k}<p_{k}^{k+1}$

The following result follows from the Firoozbakht's Conjecture.

Corollary1.1 If $p_{k}$ is the kth prime, then the for all $k \geq 1$ we have $p_{k+1}<p_{k}^{2}$.

Proof. It is easily seen that if Firoozbakht's Conjecture is true, then for all $k \geq 1$, we have $p_{k+1}<p_{k}^{\frac{k+1}{k}} \leq p_{k}^{2}$.

Remark 1.3: It should be noted here that the Corollary1.1 can be obtained from Bertrand-Chebyshev theorem, see [2]. Indeed, since $p_{k} \geq 2$, we have $p_{k+1}<2 p_{k} \leq p_{k}^{2}$, for all $k \geq 1$.

\section{Lemma 1.1: The General Classical Arithmetic-Geometric Mean Inequality}

If $x_{1}, x_{2}, \ldots, x_{n}$ are positive real numbers, then

$\left(x_{1} x_{2} \ldots x_{n}\right)^{1 / n} \leq \frac{x_{1}+x_{2}+\ldots+x_{n}}{n}$,

with equality occurring if and only if $x_{1}=x_{2}=\ldots=x_{n}$.

\section{Inequalities for the greatest prime function}

In this section we prove several inequalities concerning the greatest prime function $[x]_{p}$.

Theorem 2.1: For any integer $k \geq 1$, and for any real number $x \geq 2$, the greatest prime function satisfies the inequality $x<[x]_{p}^{\frac{k+1}{k}}$, where $[x]_{p}=p_{k}$.

Proof. Let $p_{k}$ be the kth prime number, and let $x \in R, x \geq 2$ such that $x=p_{k}+r$, where $r<p_{k+1}-p_{k}$ and $k \geq 1$ is an integer. Then

$[x]_{p}=\left[p_{k}+r\right]_{p}>p_{k+1}^{\frac{k}{k+1}}>x^{\frac{k}{k+1}}$, which implies that $x<[x]_{p}^{\frac{k+1}{k}}$.

This proves the Theorem.

Now, we also establish some new interesting inequalities related to well-known inequalities in mathematics. First, we prove the following inequality for the primes $\left[x_{1}\right]_{p},\left[x_{2}\right]_{p}, \ldots,\left[x_{n}\right]_{p}$, which is similar to a well-known general arithmetic-geometric mean inequality. So, we call it the general arithmetic-semigeometric mean inequality. 


\section{Theorem 2.2: The General Arithmetic-Semi geometric Mean Inequality}

If $x_{1}, x_{2}, \ldots, x_{n}$ are real numbers with $x_{k} \geq 2$, for $1 \leq k \leq n$, then

$$
\left(\left[x_{1} x_{2} \ldots x_{n}\right]_{p}\right)^{\frac{1}{2 n}}<\frac{\left[x_{1}\right]_{p}+\left[x_{2}\right]_{p}+\ldots+\left[x_{n}\right]_{p}}{n} .
$$

Proof. Applying Corollary1.1, let us estimate

$$
\begin{aligned}
\left(\left[x_{1} x_{2} \ldots x_{n}\right]_{p}\right)^{\frac{1}{2 n}} & \leq\left(x_{1} x_{2} \ldots x_{n}\right)^{\frac{1}{2 n}}<\left(\left[x_{1}\right]_{p}^{2} \cdot\left[x_{2}\right]_{p}^{2} \ldots\left[x_{n}\right]_{p}^{2}\right)^{\frac{1}{2 n}} \\
& =\left(\left[x_{1}\right]_{p} \cdot\left[x_{2}\right]_{p} \ldots\left[x_{n}\right]_{p}\right)^{\frac{1}{n}},
\end{aligned}
$$

from which it follows that

$$
\left(\left[x_{1} \cdot x_{2} \ldots x_{n}\right]_{p}\right)^{\frac{1}{2 n}}<\left(\left[x_{1}\right]_{p} \cdot\left[x_{2}\right]_{p} \ldots\left[x_{n}\right]_{p}\right)^{\frac{1}{n}}
$$

By using the classical arithmetic-geometric mean inequality for the prime numbers $\left[x_{1}\right]_{p},\left[x_{2}\right]_{p}, \ldots,\left[x_{n}\right]_{p}$, we get $\left(\left[x_{1} \cdot x_{2} \ldots x_{n}\right]_{p}\right)^{\frac{1}{2 n}}<\frac{\left[x_{1}\right]_{p}+\left[x_{2}\right]_{p}+\ldots+\left[x_{n}\right]_{p}}{n}$,

which completes the proof.

Corollary 2.1 If $x_{1}, x_{2}, \ldots, x_{n}$ are real numbers with $x_{k} \geq 2$, for $1 \leq k \leq n$, then

$$
\left(\left[x_{1} x_{2} \ldots x_{n}\right]_{p}\right)^{\frac{1}{n}}<\frac{\left[x_{1}\right]_{p}^{2}+\left[x_{2}\right]_{p}^{2}+\ldots+\left[x_{n}\right]_{p}^{2}}{n}
$$

Proof. This follows from the General Arithmetic-Semigeometric Mean Inequality, and from the inequality [see 1] :

$$
\frac{\left[x_{1}\right]_{p}+\left[x_{2}\right]_{p}+\ldots+\left[x_{n}\right]_{p}}{n} \leq \sqrt{\frac{\left[x_{1}\right]_{p}^{2}+\left[x_{2}\right]_{p}^{2}+\ldots+\left[x_{n}\right]_{p}^{2}}{n}} .
$$

To improve the previous estimation obtained in the Theorem 2.2, we establish the following inequality.

\section{Theorem 2.3: The General Semi-arithmetic-Geometric Mean Inequality}

If $x_{1}, x_{2}, \ldots, x_{n}$ are real numbers with $x_{k} \geq 2$, for $1 \leq k \leq n$, then

$$
\left(\left[x_{1} x_{2} \ldots x_{n}\right]_{p}\right)^{\frac{1}{n}}<\frac{\left[x_{1}\right]_{p}+\left[x_{2}\right]_{p}+\ldots+\left[x_{n}\right]_{p}}{n / 2}
$$

Proof. Using Bertrand-Chebyshev theorem, we obtain $x<p_{k+1}<2 p_{k}=2[x]_{p}$ for any real $x$.

Therefore, we get the estimate 


$$
\begin{aligned}
\left(\left[x_{1} x_{2} \ldots x_{n}\right]_{p}\right)^{\frac{1}{n}} & \leq\left(x_{1} x_{2} \ldots x_{n}\right)^{\frac{1}{n}}<\left(2^{n}\left(\left[x_{1}\right]_{p} \cdot\left[x_{2}\right]_{p} \ldots\left[x_{n}\right]_{p}\right)\right)^{\frac{1}{n}} \\
& =2\left(\left[x_{1}\right]_{p} \cdot\left[x_{2}\right]_{p} \ldots\left[x_{n}\right]_{p}\right)^{\frac{1}{n}}
\end{aligned}
$$

from which it follows that

$$
\left(\left[x_{1} x_{2} \ldots x_{n}\right]_{p}\right)^{\frac{1}{n}}<2\left(\left[x_{1}\right]_{p} \cdot\left[x_{2}\right]_{p} \ldots\left[x_{n}\right]_{p}\right)^{\frac{1}{n}}
$$

Now, using the classical arithmetic-geometric mean inequality for the prime numbers $\left[x_{1}\right]_{p},\left[x_{2}\right]_{p}, \ldots,\left[x_{n}\right]_{p}$, we get

$$
\left(\left[x_{1} x_{2} \ldots x_{n}\right]_{p}\right)^{\frac{1}{n}}<\frac{\left[x_{1}\right]_{p}+\left[x_{2}\right]_{p}+\ldots+\left[x_{n}\right]_{p}}{n / 2}
$$

which completes the proof.

Example 2.1 Consider the numbers $x_{1}=489, x_{2}=\sqrt{675}, x_{3}=\sqrt[3]{8745}, x_{4}=17 \pi$.

We find that

$$
\left[x_{1} \cdot x_{2} \cdot x_{3} \cdot x_{4}\right]_{p}^{1 / 8}=[489 \cdot \sqrt{675} \cdot \sqrt[3]{8745} \cdot 17 \pi]_{p}^{1 / 8}=[822300.503]_{p}^{1 / 8}=(822299)^{1 / 8}=5.487
$$

And

$$
\frac{[489]_{p}+[\sqrt{675}]_{p}+[\sqrt[3]{8745}]_{p}+[17 \pi]_{p}}{4}=\frac{487+23+19+53}{4}=\frac{582}{4}=145.5
$$

Example 2.2 Let $x_{1}=3.9, x_{2}=3.99, x_{3}=3.999$.

For the prime number $\left[x_{1} x_{2} \cdot x_{3}\right]_{p}$, we have

$\left[x_{1} x_{2} x_{3}\right]_{p}=[3.9 \cdot 3.99 \cdot 3.999]_{p}=[62.228]_{p}=61$,

and

$$
\left(\frac{[3.9]_{p}+[3.99]_{p}+[3.999]_{p}}{3}\right)^{6}=\left(\frac{3+3+3}{3}\right)^{6}=729
$$

Now, we use the semi-arithmetic-geometric mean inequality of type to estimate the number $\left[x_{1} \cdot x_{2} \cdot x_{3}\right]_{p}$.

We have

$$
61=\left[x_{1} \cdot x_{2} \cdot x_{3}\right]_{p}<\left(\frac{[3.9]_{p}+[3.99]_{p}+[3.999]_{p}}{3 / 2}\right)^{3}=\left(\frac{18}{3}\right)^{3}=216 .
$$

Theorem 2.4: Triangle Inequality of Type I If $x_{1}, x_{2}, \ldots, x_{n}$ are real numbers with $x_{k} \geq 2$, for $1 \leq k \leq n$, then

$$
\left(\left[x_{1}+x_{2}+\ldots+x_{n}\right]_{p}\right)^{1 / 2}<\left[x_{1}\right]_{p}+\left[x_{2}\right]_{p}+\ldots+\left[x_{n}\right]_{p}
$$

Proof. Using Corollary1.1, we get the estimation 


$$
\left[x_{1}+x_{2}+\ldots+x_{n}\right]_{p} \leq x_{1}+x_{2}+\ldots+x_{n}<\left[x_{1}\right]_{p}^{2}+\left[x_{2}\right]_{p}^{2}+\ldots+\left[x_{n}\right]_{p}^{2} \leq\left(\left[x_{1}\right]_{p}+\left[x_{2}\right]_{p}+\ldots+\left[x_{n}\right]_{p}\right)^{2}
$$

Taking the second root in the previous inequality, we get (2).

Example 2.3 Consider the real numbers $x_{1}=\sqrt{189}, x_{2}=\sqrt{275}, x_{3}=\sqrt[3]{9347}$.

We find that

$$
\left(\left[x_{1}+x_{2}+x_{3}\right]_{p}\right)^{1 / 2}=\left([\sqrt{189}+\sqrt{275}+\sqrt[3]{9347}]_{p}\right)^{1 / 2}=\left([51.395]_{p}\right)^{1 / 2}=47^{1 / 2}=6.855
$$

And

$$
\left[x_{1}\right]_{p}+\left[x_{2}\right]_{p}+\left[x_{3}\right]_{p}=[\sqrt{189}]_{p}+[\sqrt{275}]_{p}+[\sqrt[3]{9347}]_{p}=13+13+19=45
$$

Now, we will show that using Bertrand-Chebyshev theorem we can improve the previous estimation in the following inequality.

Theorem 2.5: Triangle Inequality of Type II If $x_{1}, x_{2}, \ldots, x_{n}$ are real numbers with $x_{k} \geq 2$, for $1 \leq k \leq n$, then

$$
\frac{1}{2}\left[x_{1}+x_{2}+\ldots+x_{n}\right]_{p}<\left(\left[x_{1}\right]_{p}+\left[x_{2}\right]_{p}+\ldots+\left[x_{n}\right]_{p}\right) .
$$

Proof. For any real number $x$, there is $k \in \mathrm{Z}^{+}$such that $x=p_{k}+r_{k}$, where $p_{k}=[x]_{p}, r_{k}<p_{k+1}-p_{k}$.

Using Bertrand-Chebyshev theorem we obtain $x<p_{k+1}<2 p_{k}=2[x]_{p}$, for any real $\mathrm{x}$.

Then, we get the estimation

$$
\left[x_{1}+x_{2}+\ldots+x_{n}\right]_{p} \leq x_{1}+x_{2}+\ldots+x_{n}<2\left(\left[x_{1}\right]_{p}+\left[x_{2}\right]_{p}+\ldots+\left[x_{n}\right]_{p}\right) \text {. }
$$

Example 2.4 Let $x_{1}=\sqrt{189}, x_{2}=\sqrt{275}, x_{3}=\sqrt[3]{9347}$.

First, Let us estimate the prime number $\left[x_{1}+x_{2}+x_{3}\right]_{p}$ using the triangle Inequality of Theorem 2.4

$$
\left[x_{1}+x_{2}+x_{3}\right]_{p}=[\sqrt{189}+\sqrt{275}+\sqrt[3]{9347}]_{p}=\left([51.395]_{p}\right)=47
$$

and

$$
\left(\left[x_{1}\right]_{p}+\left[x_{2}\right]_{p}+\left[x_{3}\right]_{p}\right)^{2}=\left([\sqrt{189}]_{p}+[\sqrt{275}]_{p}+[\sqrt[3]{9347}]_{p}\right)^{2}=(13+13+19)^{2}=2025 .
$$

Now, we use the triangle inequality Theorem 2.5 to estimate the prime number $\left[x_{1}+x_{2}+x_{3}\right]_{p}$, and obtain

$$
\left[x_{1}+x_{2}+x_{3}\right]_{p}=[51.395]_{p}=47
$$

On the other hand, we have

$$
2\left(\left[x_{1}\right]_{p}+\left[x_{2}\right]_{p}+\left[x_{3}\right]_{p}\right)=2\left([\sqrt{189}]_{p}+[\sqrt{275}]_{p}+[\sqrt[3]{9347}]_{p}\right)=2(13+13+19)=90
$$


Theorem 2.6: Inequality of Bernoulli Type. If $x$ is a real number with $x \geq 2$, then

$\left(1+[x]_{p}\right)^{n} \geq 1+n x$, for any integer $n \geq 2$,

where $[x]_{p}$ is the greatest prime number of real $x$, and $[x]_{p}=p_{s}$ is sth prime number.

Proof. Let $[x]_{p}$ be any prime number, and let $x=p_{s}+r_{s}$, where $r_{s}<p_{s+1}-p_{s}$.

The proof utilizes mathematical induction. The case $n=2$ yields inequality

$$
\left(1+[x]_{p}\right)^{2}=\left(1+\left[p_{s}+r\right]_{p}\right)^{2}=\left(1+p_{s}\right)^{2}=1+2 p_{s}+p_{s}^{2}
$$

Using the Corollary 1.1, and Bertrand-Chebyshev theorem we get

$$
\left(1+[x]_{p}\right)^{2}>1+2 p_{s}+p_{s}^{2}>1+2 p_{s+1}>1+2 x
$$

So that the assertion is valid in this case. Thus, we assume the validity of the inequality (3) for a positive $n$, and shall deduce it for $n+1$. The assumption of $\left(1+[x]_{p}\right)^{n}>1+n x$, and the fact that $1+[x]_{p}>0$ implies that

$$
\begin{aligned}
\left(1+[x]_{p}\right)^{n+1} & >(1+n x)\left(1+[x]_{p}\right)=1+n x+[x]_{p}(1+n x) \\
& >1+n x+n x[x]_{p} \\
& \geq 1+n x+2 n x, \text { since }[x]_{p} \geq 2 . \\
& >1+n x+x=1+(n+1) x .
\end{aligned}
$$

Thus, the inequality (3) for $n+1$ follows. Hence the inequality is true for all integers $n \geq 2$.

Example 2.5 Let $x_{1}=\sqrt{5}$. Then $\left(1+[\sqrt{5}]_{p}\right)^{2}=3^{2}=9 \geq 1+2 * \sqrt{5}=5.472$.

\section{Conclusion}

Here, we present a new function $f(x)=[x]_{p}, x \in R$, with $\mathrm{x} \geq 2$, which we call the greatest prime function. Because our results could be applied in the theory of prime numbers and other fields of science, they are likely to be of great interest to the researchers.

\section{References}

[1] P. S. Bullen, D. S., Mitrinovic and P. M. Vasic, Means and Their Inequalities, Dordrecht, Holland: D. Reidel Publishing Company, (1988). http://dx.doi.org/10.1007/978-94-017-2226-1.

[2] R. Crandall, C. Pomerance, Prime numbers: A computational perspective, Springer, New York, (2001). http://dx.doi.org/10.1007/978-1-46849316-0.

[3] P. Ribenboim, the Little Book of Bigger Primes, Second Edition, Springer-Verlag, New York, Inc (2004).

[4] J. Sandor, "On Certain Bounds and Limits for Prime Numbers," Notes on Number Theory and Discrete Mathematics 18(1), (2012). 